POLARIZABILIDADES ELETRÔNICAS DOS MALEATOS DE NEODÍMIO E ÉRBIO HIDRATADOS

\title{
ELECTRONIC POLARIZABILITIES OF NEODYMIUM AND ERBIUM MALEATES HYDRATED
}

\author{
LIMA, Francisco José Santos ${ }^{1 *}$; MELO, Roseane Maria de ${ }^{1}$; SILVA, Ademir Oliveira da ${ }^{1}$. \\ ${ }^{1}$ Universidade Federal do Rio Grande do Norte \\ Centro de Ciências Exatas e da Terra - Instituto de Química \\ e-mail: limafjs@yahoo.com
}

Received 20 July 2011; received in revised form 03 August 2011; accepted 09 August 2011

\begin{abstract}
RESUMO
A força do oscilador e a polarizabilidade eletrônica têm sido usadas para avaliar modificações nos níveis de energia de íons metálicos nos compostos de coordenação. A primeira propriedade avalia a perturbação nos estados de energia causada pela força do campo ligante dos grupos de átomos que se encontram ligados com o metal. A segunda propriedade avalia a facilidade na qual o campo elétrico da radiação promove uma transição eletrônica. Neste trabalho foram avaliadas estas propriedades para os maleatos de neodímio e de érbio hidratados. De acordo com os resultados, foi possível observar que há um significativo aumento na deformação da nuvem eletrônica do íon $\mathrm{Nd}^{3+}$, na transição de menor energia. No maleato de érbio também foi observado um aumento na polarizabilidade, na transição de menor energia. Isto nos leva a concluir que, nestes sistemas, a região espectral de baixa energia influenciou mais na deformação da nuvem eletrônica das espécies.
\end{abstract}

Palavras-chave: Compostos de Coordenação, Lantanídeos, Espectroscopia.

\begin{abstract}
The oscillator strength and the polarizability electronic have been used to evaluate modifications in the levels of energy of metallic ions in the coordination compound. The first property evaluates the disturbance in the states of energy caused by the force of the ligand field of the groups of atoms that are linked with the metal. The second property evaluates the easiness in which the electric field of the radiation promotes an electronic transition. In this work they were appraised these properties for the neodymium and erbium hydrated maleates. In agreement with the results, it was possible to observe that there is a significant increase in the deformation of the electronic cloud of the ion $\mathrm{Nd} 3+$, in the transition of smaller energy. In the erbium maleate an increase was also observed in the polarizability, in the transition of smaller energy.. We ended that, in these systems, the spectral area of low energy influenced more in the deformation of the electronic cloud of the species.
\end{abstract}

Keywords: Coordination Compounds, Lanthanides, Spectroscopy

PERIÓDICO TCHÊ QUÍMICA • www.periodico.tchequimica.com • Vol. 8 N. 16.

- ISSN 1806-0374 (impresso) • ISSN 1806-9827 (CD-ROM) • ISSN 2179-0302 (meio eletrônico)

(C) 2011. Porto Alegre, RS. Brasil 


\section{INTRODUÇÃO}

A síntese de compostos de coordenação atualmente tem como propósito avaliar algumas propriedades físicas e químicas que possam evidenciar, em curto prazo, uma solução promissora de arranjos químicos experimentais que venham disponibilizar uma alternativa aos sistemas pesquisados em diversas áreas industriais e comerciais. Dentre as diferentes propriedades apresentadas pelos sistemas complexos, a interação com a radiação eletromagnética é uma das mais evidentes. A polarizabilidade eletrônica é compreendida como uma alteração que ocorre na distribuição dos elétrons das espécies, quando um campo eletromagnético como a luz, interage com sistemas moleculares, reorganizando sua configuração, ao causar uma transição eletrônica entre níveis de energia diferentes (Lima et al, 2007).

\section{Força do Oscilador e Polarizabilidades Eletrônicas}

Os parâmetros espectroscópicos avaliados neste trabalho foram a força do oscilador e a polarizabilidade eletrônica, que são obtidos a partir das seguintes expressões respectivamente: (Lima et al, 1996; 2007).

$$
\mathrm{P}_{\mathrm{ok}}=4,319 \times 10^{-9} \frac{9 \eta}{\left(\eta^{2}+2\right)^{2}} \cdot \int \varepsilon(\bar{V}) d \bar{\nu}
$$

e

$$
\alpha=\frac{e^{2}}{4 \pi^{2} M e} \sum \frac{P_{o k}\left(v_{o k}{ }^{2}-v^{2}\right)}{\left(v_{o k}^{2}-v^{2}\right)^{2}+4 v^{\prime 2} v^{2}}
$$

Diversos autores na literatura têm usado a equação (1) para interpretar a influência do campo ligante nos níveis de energia de íons metálicos em compostos de coordenação e associado alguns valores para justificar certas propriedades aplicativas destes sistemas moleculares (Davidenko and Lugina, 1968; Yatsimirskii and Davidenko, 1979; Sinha, 1982, Iftkhar, 1987, Khan et al., 2004). Estas propriedades têm como propósito avaliar como os sistemas que absorvem luz se comportam quando determinadas transições neles ocorrem. A primeira equação avalia a probabilidade de uma transição, e de certa forma, mede a intensidade da luz absorvida bem como a perturbação que ocorre no meio; e a segunda, o grau da distorção eletrônica causada na reorganização da configuração eletrônica quando ocorre a mudança de elétrons de um estado de energia para outro em uma molécula. As duas informações são bastante úteis para interpretar a intensidade da interação que ocorre entre os ligantes (iônico ou neutro), e o elemento que será avaliado pela transição, que nesse caso é um íon lantanídeo (neodímio ou érbio).

O objetivo desse trabalho foi de observar o comportamento das propriedades ópticas destes compostos através da força do oscilador e das polarizabilidades eletrônicas e, através destes, concluir como as espécies ligantes facilitam na promoção dos elétrons e na forma de organização dos níveis de energia nestes sistemas moleculares.

\section{PARTE EXPERIMENTAL}

As sínteses dos sais de maleatos de neodímio hidratados foram preparadas a partir dos respectivos cloretos hidratados, obtidos em etapa anterior por Melo et al. (2009), pela adição do ácido maleico em solução aquosa numa proporção 1:4 e, em sequência, secados, lavados com água destilada para retirada do excesso de ácido e posteriormente secados novamente sob vácuo, para a formação do sal, segundo o processo:

$$
\begin{gathered}
\mathrm{LnCl}_{3} \cdot \mathrm{nH}_{2} \mathrm{O}_{(\mathrm{aq})}+4 \mathrm{HMal}_{(\mathrm{aq})} \rightarrow \mathrm{Ln}(\mathrm{Mal})_{\mathrm{x} \cdot \mathrm{yH}} \mathrm{O}+ \\
3 \mathrm{HCl}_{(\mathrm{aq})}+(4-\mathrm{x}) \mathrm{HMal}_{(\mathrm{aq})} \rightarrow \\
\left(\text { banho Maria } 60^{\circ} \mathrm{C}\right)
\end{gathered}
$$$$
\mathrm{Ln}(\mathrm{Mal})_{\mathrm{x} \cdot \mathrm{yH}_{2} \mathrm{O}(\mathrm{s})}+(4-\mathrm{x}) \mathrm{HMal}_{(\mathrm{s})}+3 \mathrm{HCl}_{(\mathrm{v})} \cdot \rightarrow
$$

(lavado com água destilada, filtrado) $\rightarrow$

$$
\begin{gathered}
\text { (secado sob vácuo) } \rightarrow \\
\operatorname{Ln}(\text { Mal })_{x} \mathrm{yH}_{2} \mathrm{O}_{(\mathrm{s})}
\end{gathered}
$$

As microanálises das amostras (análise complexométrica e microanálise de $\mathrm{C}$ e $\mathrm{H}$ ), permitiram a obtenção das seguintes estequiometrias: $\mathrm{La}(\mathrm{mal})_{2} .9 \mathrm{H}_{2} \mathrm{O}, \mathrm{Nd}(\mathrm{mal})_{3} .14 \mathrm{H}_{2} \mathrm{O}$ e $\mathrm{Er}(\mathrm{mal})_{2} \cdot 16 \mathrm{H}_{2} \mathrm{O}$. Após a síntese, os compostos foram caracterizados por análise térmica, 
condutância molar, espectros na região do infravermelho, difração de raios-x e modelagem molecular, conforme descrito em MELO et al, 2009. Neste trabalho, foram calculadas as polarizabilidades eletrônicas a partir dos espectros dos sais de neodímio e érbio em solução etanólica, tal como descrito em LIMA et al, 2010. Os espectros foram registrados em um equipamento UV-visível Nicolet Evolution 100 e as seqüências dos experimentos se encontram visualizadas no fluxograma da Figura 1. A força do oscilador foi calculada mediante equação $1 \mathrm{e}$ pelo uso do programa SIMP2FOS, desenvolvido em linguagem QBASIC. As polarizabilidades foram calculadas a partir da equação 2 e pelo uso do programa POLAZ-F, desenvolvido em linguagem QBASIC. Os gráficos das polarizabilidades eletrônicas dos maleatos estão mostrados nas Figuras 2 e 3 e foram obtidos a partir dos baricentros das transições e da força do oscilador, que se encontram descritas na Tabela 1, juntamente com outras informações. Os valores de polarizabilidades estão disponibilizados na Tabela 2. Correlações de força do oscilador e polarizabilidades eletrônicas versus baricentro das transições estão mostrados na Figura 4.

\section{RESULTADOS E DISCUSSÕES}

Os espectros na região do visível dos maleatos de neodímio e de érbio apresentaram duas bandas de absorção relevantes nas regiões pesquisadas, no intervalo de 400 a $760 \mathrm{~nm}$. As transições do neodímio que se apresentaram mais intensas foram as hipersensitivas ${ }^{4} \mathrm{I}_{9 / 2} \rightarrow{ }^{4} \mathrm{G}_{7 / 2}$, ${ }^{4} G_{9 / 2}(491-544 \mathrm{~nm})$ e ${ }^{4} I_{9 / 2} \rightarrow{ }^{4} G_{5 / 2},{ }^{2} G_{7 / 2}(559-614$ $n m)$. Para o érbio foram as transições ${ }^{4} I_{15 / 2} \rightarrow{ }^{2} H_{11 / 2}$ $(510-535 \mathrm{~nm})$ e ${ }^{4} I_{15 / 2} \rightarrow{ }^{4} F_{9 / 2}(630-685 \mathrm{~nm})$, sendo a primeira hipersensitiva. A segunda transição do érbio não é considerada uma transição hipersensitiva por não se alterar muito com a mudança do ambiente químico. Ainda assim temos registrado a sua ocorrência, por se apresentar com uma intensidade comparável à primeira transição, e por isso foram avaliados os parâmetros espectrais para a mesma.

Analisando as transições para o neodímio, influenciadas pelo campo ligante do íon maleato, percebeu-se que, na transição de menor energia, ${ }^{4} I_{9 / 2} \rightarrow{ }^{4} G_{5 / 2},{ }^{2} G_{7 / 2}(559-614 \mathrm{~nm})$, os valores da força do oscilador e da polarizabilidade foram mais elevados, indicando que a influência do campo causa uma maior probabilidade na transferência do elétron entre esses níveis e a distorção da configuração é mais acentuada nesta transição hipersensitiva do que na outra transição, também hipersensitiva, registrada. Estes valores estão mostrados nas Tabelas 1 e 2 e Figuras 2 e 4 . Para o érbio, observou-se que a força do campo ligante dos íons maleatos provocou um maior valor na força do oscilador, para a transição hipersensitiva ${ }^{4} I_{15 / 2} \rightarrow{ }^{2} H_{11 / 2}$, que ocorre numa região de maior energia $(510-535 \mathrm{~nm})$. No entanto, o mesmo aumento não foi observado para a polarizabilidade eletrônica nesta transição. Para a polarizabilidade, o maior valor observado foi para a transição não hipersensitiva ${ }^{4} \mathrm{I}_{15 / 2} \rightarrow{ }^{4} \mathrm{~F}_{9 / 2}$, que ocorre numa faixa de energia mais baixa, no intervalo de comprimentos de onda de 630 a 685 $\mathrm{nm}$, como mostram as Tabelas 1 e 2 e Figuras 3 e 4. Uma possível explicação para a inversão no caso do composto de érbio deve-se ao fato de a força do oscilador, na transição hipersensitiva que ocorre numa região de maior energia, não possuir uma probabilidade relevante a ponto de compensar o valor do conteúdo energético para o deslocamento do elétron entre os níveis envolvidos. Já na transição não-hipersensitiva, a força do oscilador é menor, como esperado, mas apesar de ocorrer com uma menor probabilidade, esse efeito é compensado pelo menor gasto energético, originando por isso uma maior polarizabilidade. Em termos práticos, a eficiência da transferência de elétrons é maior, pois é necessário menos energia para provocar uma reorganização eletrônica no sistema.

Foi observado também que o efeito do potencial iônico apresenta uma correlação direta com o comportamento das transições e das propriedades espectrais observadas. Nas transições de menor energia, percebeu-se que, para o neodímio, apresenta força do oscilador e polarizabilidades eletrônicas maiores que para o érbio, como pode-se observar nas Tabelas 1 e 2 e nas Figuras 2, 3 e 4. Para as transições de maior energia, o érbio apresentou maior força do oscilador e maior polarizabilidade eletrônica. Isto nos leva a crer que o maior potencial iônico do érbio induz na transição de maior energia, uma maior probabilidade de transição e uma maior deformação na nuvem eletrônica do érbio. 


\section{CONCLUSÕES}

De acordo com os valores obtidos, foi possível observar que há um significativo aumento na deformação da nuvem do íon $\mathrm{Nd}^{3+}$ na segunda transição em relação ao maleato de érbio. Isto mostra que, nestes sistemas, apenas o aumento no caráter covalente da ligação (« oscila entre 0,100 e 1,620 para o $\mathrm{Nd}^{3+}$ e na faixa de 0,200 para o $\mathrm{Er}^{3+}$ ) (Lima et al., 2010), não justifica necessariamente um aumento de polarizabilidade nos íons. Percebe-se, principalmente, que é fundamental que a região espectral do elétron óptico proporcione um efeito de probabilidade de transição interessante, e que a força do oscilador, que é função do coeficiente de absorção integrado, contribui significativamente para isso. Neste sentido, acredita-se que a polarizabilidade eletrônica pode ser aplicada no acompanhamento da evolução dos estados eletrônicos intermediários da transição, sendo compreendida como uma medida da facilidade com que a nuvem eletrônica de um átomo pode ser distorcida, em função do efeito da interação da radiação eletromagnética com sistemas químicos possibilitando dessa forma, uma melhor avaliação na reorganização eletrônica para direcionar uma melhor aplicação dos mesmos.

\section{AGRADECIMENTOS}

Ao PIBIC / UFRN / PROPESQ / CNPq, pelo incentivo à pesquisa em química fundamental, e ao laboratório NEPGN (espectros na região visível).

\section{REFERÊNCIAS:}

1. Davidenko, N. K. And Lugina, L. N.; 1968 - Spectra and Structure of the Aquo-ions of Neodymium, Europium, and Erbium Russ. J. Inorg. Chem., 13, 512-17.

2. Iftkhar, k. 1987- Hypersensitivity in the 4 f$4 \mathrm{f}$ absorption spectra of lanthanide(III) complexes - Inorg. Chim. Acta, 129, 261-
264.

3. Khan, A. A., Hussain, H. A., And Iftikhar, K., 2004 - 4f-4f Absorption Spectra And Hypersensitivity In Nine-Coordinate $\mathrm{Ho}$ (III) And Er(III) Complexes In Different Environments - Spect. Acta Part A 60, 2087-2092.

4. Lima, F. J. S.; Brito, H. F.; Silva, A. G.; Silva, A. O; Braga, C. C. M; Lima, A. J. P; Cardoso, M. C. C. 1996 - O Uso da Força do Oscilador na Avaliação de Intensidades Espectrais - Anais da Associação Brasileira de Química, 45; (1), 31-35.

5. Lima, F. J. S.; Silva, A. G.; Silva, A. O.; Santos, J. E., 2007 - Estudos Espectroquímicos de Cloretos Hiidratados de Samário e Disprósio e seus Complexos com Acetato de Etila Revista Tchê-Química, 04, 31-37.

6. Lima, F. J. S., Melo, R. M., Lima, F. J. S., Silva, A. O. E Braga, C. C. M., 2010 Parâmetros de Ligação e Força do Oscilador dos Maleatos de Neodímio e Érbio Hidratados - Revista Tchê-Química, $07,14,52-56$.

7. Melo, R. M., Lima, F. J. S., Silva, A. O. E Braga, C. C. M., 2009 - Síntese e Caracterização dos Maleatos de Lantânio, Neodímio e Érbio. - Revista TchêQuímica, 06, 11, 31-42.

8. Sinha, S. P., 1982 - Some Spectroscopic Aspects of the Lanthanides - An. Acad. Ciênc. do Estado de São Paulo, 36, 1-71.

9. Yatsimirskii, K. B. And Davidenko, N. 1979 - Absorption Spectra and Structure Of Lanthanide Coordination Compounds in Solution - Coord. Chem. Rev, 27, 223273. 


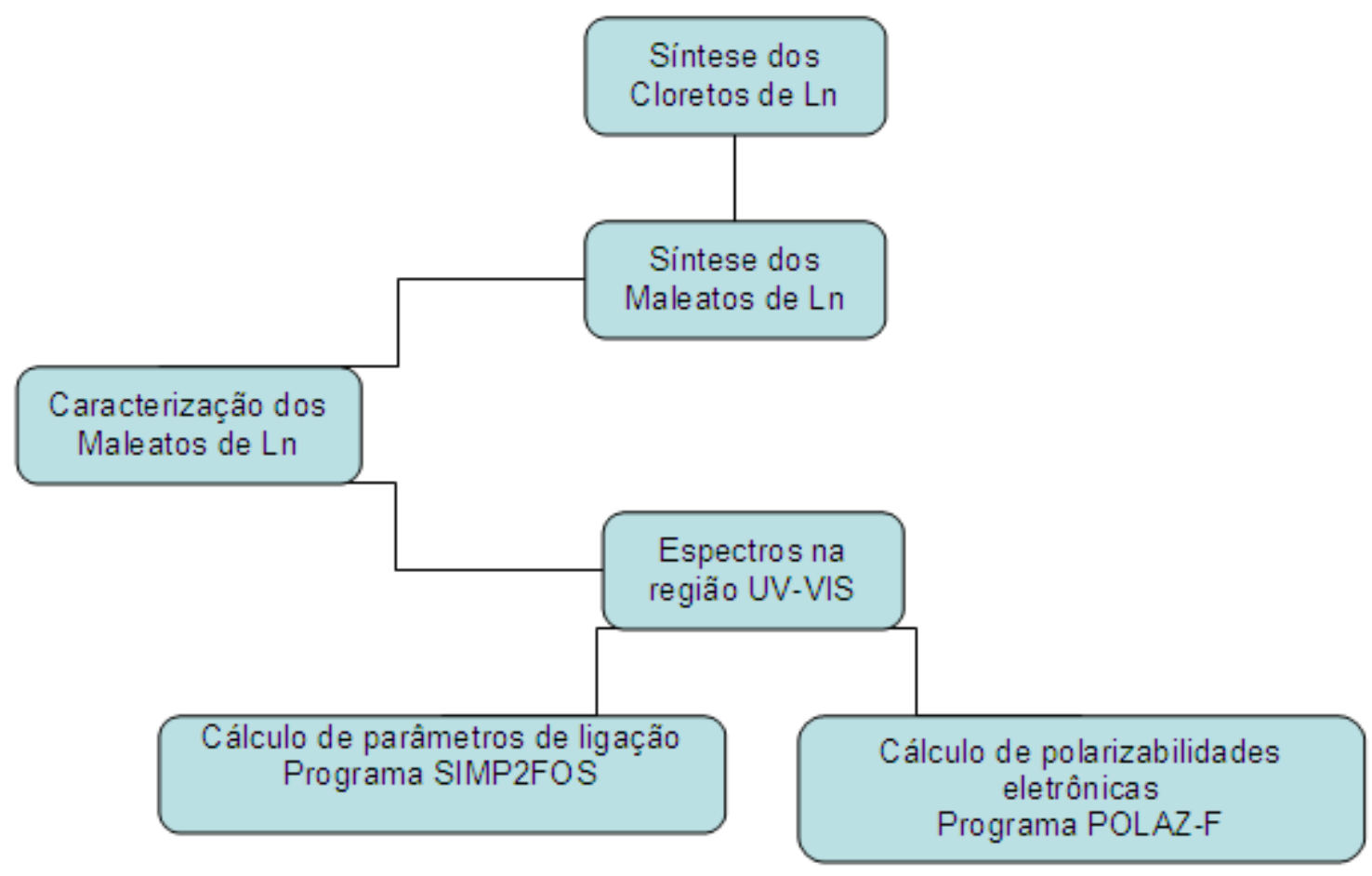

Figura 1. Fluxograma da ordem dos experimentos

Tabela 1. Dados experimentais das transições pesquisadas para os maleatos de neodímio e érbio.

\begin{tabular}{|c|c|c|c|c|}
\hline \multirow[b]{2}{*}{ Faixa (nm) } & \multicolumn{2}{|c|}{ Neodímio } & \multicolumn{2}{|c|}{ Érbio: } \\
\hline & $491-544$ & $559-614$ & $510-535$ & $630-685$ \\
\hline Transição & ${ }^{4} I_{9 / 2} \rightarrow{ }^{4} \mathrm{G}_{7 / 2},{ }^{4} \mathrm{G}_{9 / 2}$ & ${ }^{4} I_{9 / 2} \rightarrow{ }^{4} G_{5 / 2},{ }^{2} G_{7 / 2}$ & ${ }^{4} \mathrm{I}_{15 / 2} \rightarrow{ }^{2} \mathrm{H}_{11 / 2}$ & ${ }^{4} \mathrm{I}_{15 / 2} \rightarrow{ }^{4} \mathrm{~F}_{9 / 2}\left({ }^{*}\right)$ \\
\hline Baricentro da transição $\left(\mathrm{cm}^{-1}\right)$ & 19341,21 & 17069,74 & 19148,06 & 15244,45 \\
\hline $\begin{array}{l}\text { Baricentro da transição } \\
\left(\times 10^{14} \text { Hertz }\right)\end{array}$ & 5,798 & 5,118 & 5,741 & 4,570 \\
\hline Força do oscilador $\left(\times 10^{-6}\right)$ & 3,856 & 7,966 & 6,594 & 4,952 \\
\hline
\end{tabular}


Tabela 2. Dados experimentais da polarizabilidade a partir dos valores da frequência da transição e da força do oscilador.

\begin{tabular}{|c|c|c|c|c|}
\hline \multirow[b]{2}{*}{ Transição $\left(^{*}\right)$} & \multicolumn{2}{|c|}{ Neodímio } & \multicolumn{2}{|c|}{ Érbio } \\
\hline & $1^{a}$ & $2^{\mathrm{a}}$ & $1^{a}$ & $\left.2^{\mathrm{a}(* *}\right)$ \\
\hline Polarizabilidade Estática $\left(\times 10^{-28} \mathrm{~cm}^{3}\right)$ & 0,734 & 1,953 & 1,285 & 1,523 \\
\hline Polarizabilidade Máxima $\left(\times 10^{-21} \mathrm{~cm}^{3}\right)$ & 1,610 & 4,836 & 2,836 & 4,223 \\
\hline Polarizabilidade Mínima $\left(\times 10^{-21} \mathrm{~cm}^{3}\right)$ & $-1,610$ & $-4,836$ & $-2,836$ & $-4,223$ \\
\hline Polarizabilidade Fora de Fase $\left(\times 10^{-21} \mathrm{~cm}^{3}\right)$ & 3,220 & 9,673 & 5,673 & 8,45 \\
\hline
\end{tabular}

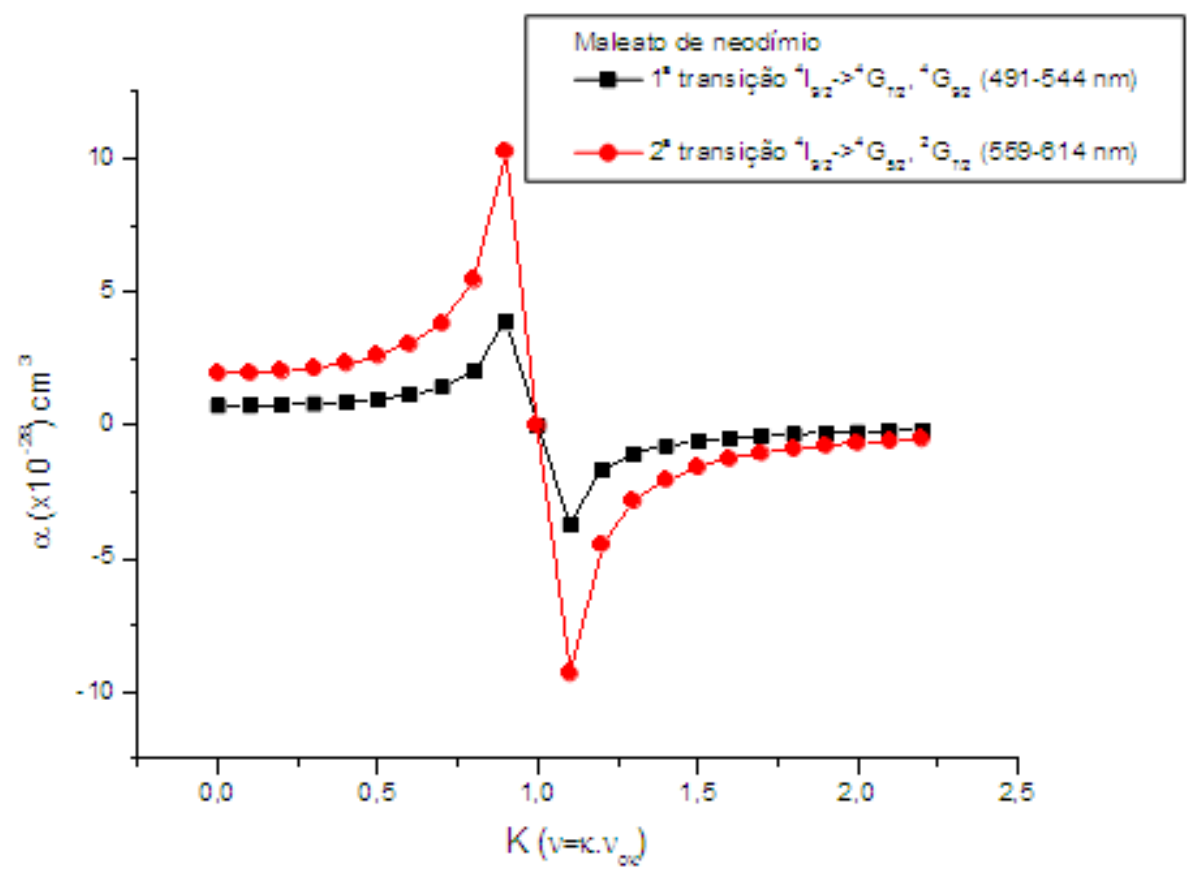

Figura 2. Polarizabilidades do Neodímio ( $1^{a}$ e $2^{a}$ transição) 


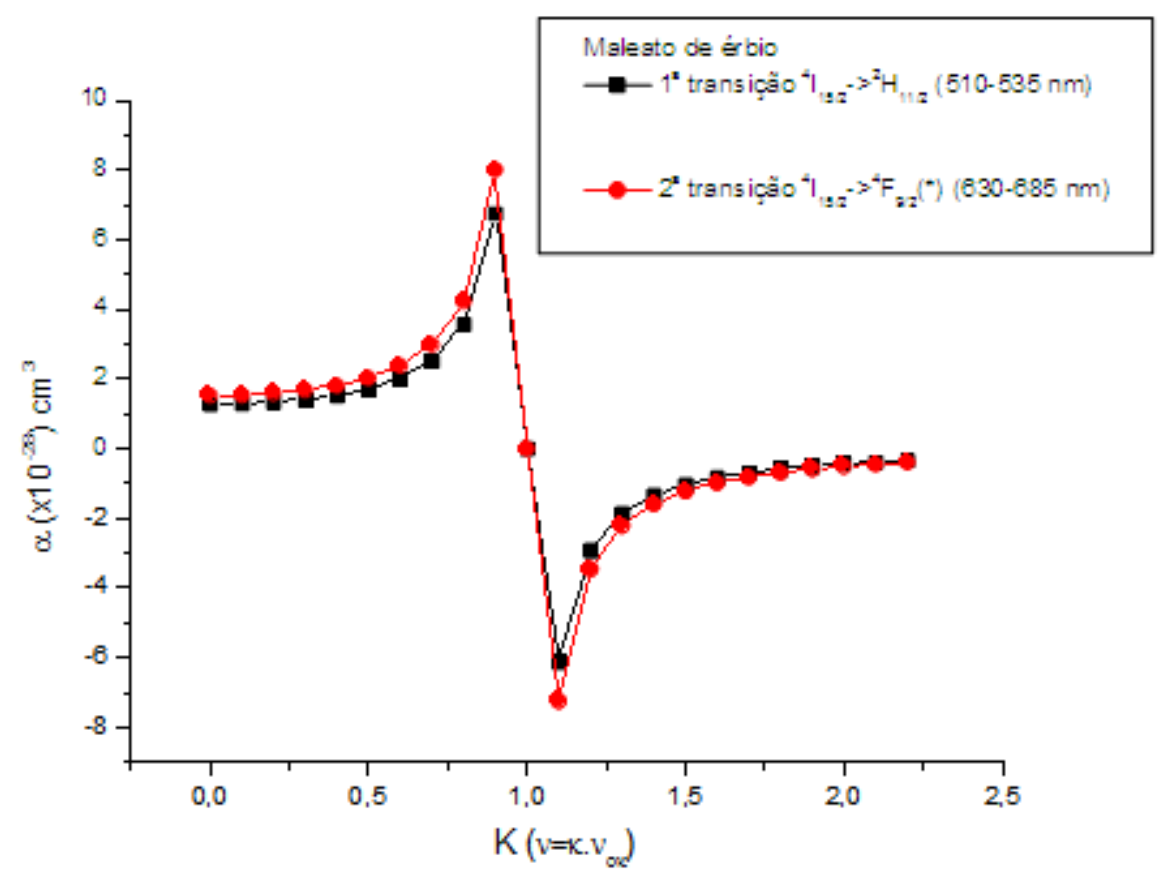

Figura 3. Polarizabilidades do Érbio ( $1^{a}$ e $2^{a}$ transição).

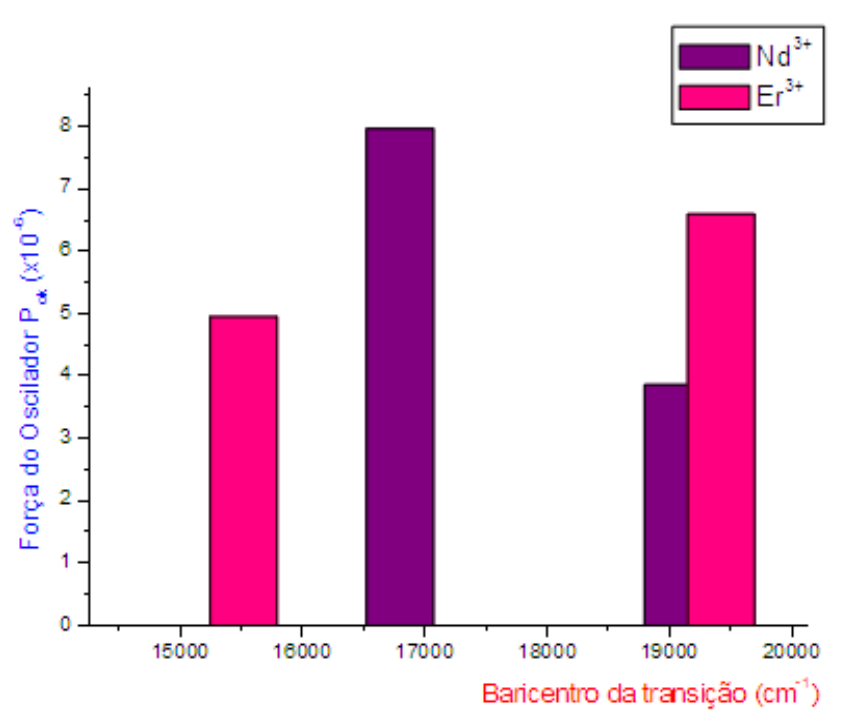

(a)

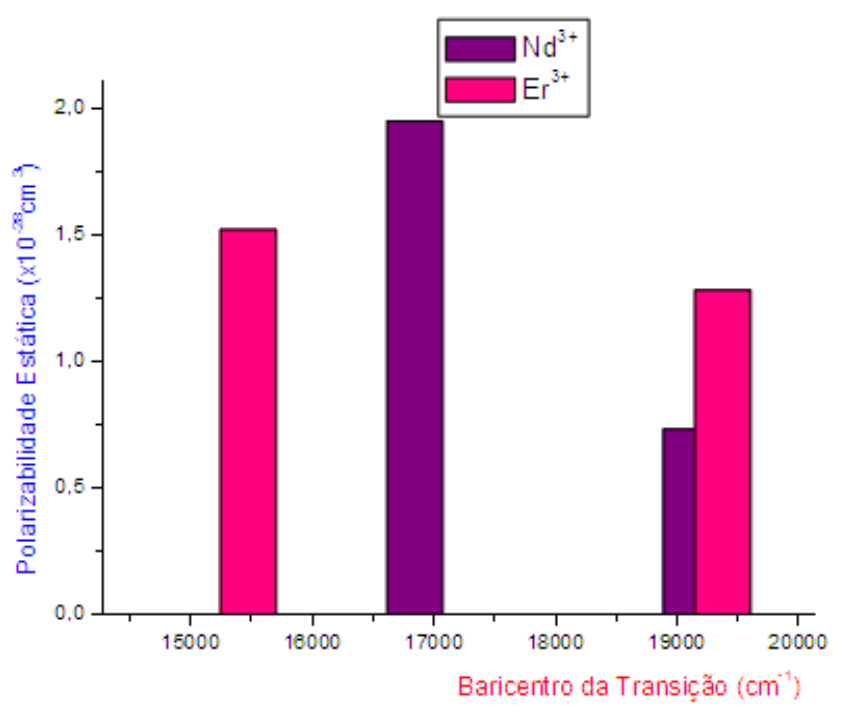

(b)

Figura 4. Influência da força do campo ligante do íon maleato nas transições pesquisadas dos íons $\mathrm{Nd}^{3+}$ e $\mathrm{Er}^{3+}$, avaliadas pela força do oscilador (a) e polarizabilidade eletrônica (b).

PERIÓDICO TCHÊ QUÍMICA • www.periodico.tchequimica.com • Vol. 8 N. 16.

- ISSN 1806-0374 (impresso) • ISSN 1806-9827 (CD-ROM) • ISSN 2179-0302 (meio eletrônico)

(C) 2011. Porto Alegre, RS. Brasil

The Periódico Tchê Quimica (ISSN: 1806-0374; 2179-0302) is an open-access journal since 2004. Journal DOI: 10.52571/PTQ. http://www.tchequimica.com. This text was introduced in this file in 2021 for compliance reasons.

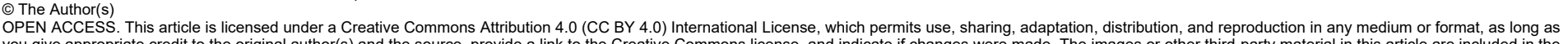
you give appropriate credit to the original author(s) and the source, provide a link to the Creative Commons license, and indicate if changes were made. The images or other third-party material in this article are included in the or exceeds the permitted use, you will need to obtain permission directly from the copyright holder. To view a copy of this license, visit http://creativecommons org/licenses/by/4.0l. or exceeds the permitted use, you will need to obtain permission directly from the copyright holder. To view a copy of this license, visit http://creativecommons.org/licenses/by/4.0/. 